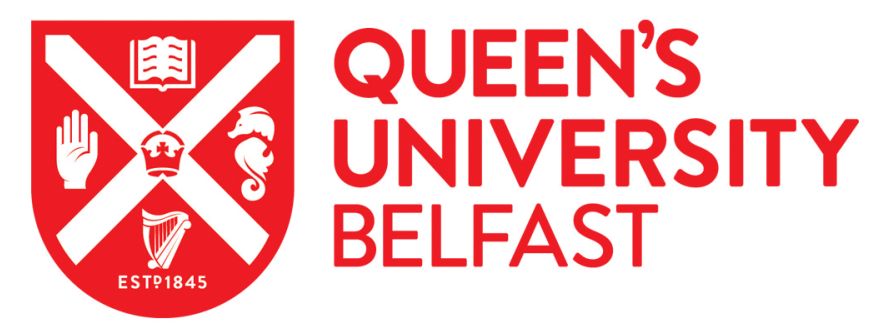

\title{
Trauma-informed care: A qualitative study exploring the views and experiences of professionals in specialist health services for adults with intellectual disabilities
}

Truesdale, M., Brown, M., Taggart, L., Bradley, A., Paterson, D., Sirisena, C., Walley, R., \& Karatzias, T. (2019). Trauma-informed care: A qualitative study exploring the views and experiences of professionals in specialist health services for adults with intellectual disabilities. Journal of Applied Research in Intellectual Disabilities. https://doi.org/10.1111/jar.12634

Published in:

Journal of Applied Research in Intellectual Disabilities

Document Version:

Peer reviewed version

Queen's University Belfast - Research Portal:

Link to publication record in Queen's University Belfast Research Portal

Publisher rights

() 2019 John Wiley \& Sons Ltd. This work is made available online in accordance with the publisher's policies. Please refer to any applicable terms of use of the publisher.

\section{General rights}

Copyright for the publications made accessible via the Queen's University Belfast Research Portal is retained by the author(s) and / or other copyright owners and it is a condition of accessing these publications that users recognise and abide by the legal requirements associated with these rights.

\section{Take down policy}

The Research Portal is Queen's institutional repository that provides access to Queen's research output. Every effort has been made to ensure that content in the Research Portal does not infringe any person's rights, or applicable UK laws. If you discover content in the

Research Portal that you believe breaches copyright or violates any law, please contact openaccess@qub.ac.uk. 


\title{
$\underline{\text { Title }}$
}

Trauma-informed care: A qualitative study exploring the views and experiences of professionals in specialist health services for adults with intellectual disabilities

\author{
Authors \\ Maria Truesdale ${ }^{1}$ \\ Michael Brown ${ }^{2}$ \\ Laurence Taggart ${ }^{3}$ \\ Aoife Bradley ${ }^{1}$ \\ Douglas Paterson ${ }^{4}$ \\ Chammy Sirisena ${ }^{5}$ \\ Robert Walley ${ }^{1}$ \\ Thanos Karatzias ${ }^{1,6}$
}

\section{$\underline{\text { Affiliations }}$}

${ }^{1}$ Edinburgh Napier University, School of Health and Social care, Edinburgh, UK

${ }^{2}$ Queen's University Belfast, School of Nursing \& Midwifery, Belfast, UK

${ }^{3}$ Ulster University, School of Nursing, Northern Ireland, UK

${ }^{4}$ NHS Ayrshire and Arran, Kilmarnock, UK

${ }^{5}$ NHS Norders, Earlston, UK

${ }^{6}$ NHS Lothian, Rivers centre for Traumatic Stress, Edinburgh, UK

\section{Address for correspondence}

Dr Maria Truesdale

Edinburgh Napier University

Sighthill Campus, Sighthill Court

Edinburgh, EH11 4BN. Tel. (+44) (0) 1314552663

Email: m.truesdale@napier.ac.uk 


\title{
$\underline{\text { Title }}$
}

Trauma-informed care: A qualitative study exploring the views and experiences of professionals in specialist health services for adults with intellectual disabilities

\begin{abstract}
Background: Although, trauma and its sequelae is increasingly recognised as a major morbidity factor in people with intellectual disabilities, there has been a lack of inquiry into how health care professionals address trauma in adults with ID.

Aims: This study aimed to explore the perspectives of practitioners in specialist intellectual disability health services involved in the assessment and treatment of adults with intellectual disability regarding the current provision and the necessary developments to address trauma in adults with intellectual disabilities.

Methods: Twenty-five face-to-face and telephone qualitative interviews involving practitioners from specialist intellectual disability health services were conducted across 6 different geographical health service areas in the United Kingdom. Data were analysed using thematic content analysis.

Findings: Analysis of the data revealed seven central themes: (i) unmasked trauma; (ii) trauma informed care; (iii) person-centred care and support; (iv) multi-disciplinary working; (v) reasonable adjustments; (vi) barriers to treatment and (vii) awareness, training and education.

Conclusion and Implications: The study highlighted the need for trauma-informed care and multi-disciplinary working as key features for future service development. It also recognised the urgent need to develop an evidence-base for effective psychological interventions for PTSD in adults with ID in addition to the need for awareness, training and education of health care staff, in order to improve service provision amongst this population group.
\end{abstract}

Keywords: intellectual disabilities, trauma, qualitative, health care professionals 


\section{Introduction}

DSM-5 Post Traumatic Stress Disorder (PTSD) (DSM-5; American Psychiatric Association, 2013) is common in the general population as well as in people with Intellectual Disabilities (IDs), with prevalence rates of $60 \%$ cited in some studies (Gilderthorp, 2015). PTSD is a debilitating condition in response to a traumatic life event and includes 20 symptoms that are organized under four symptom clusters; intrusions, avoidance, negative alterations in cognitions and mood, and alternations in arousal and reactivity (APA, 2013). There is evidence to suggest that people with IDs are more likely to be exposed to traumatic life events compared to the general population (Horner-Johnson and Drum, 2006; Hughes et al., 2012; Jones et al., 2012; Leeb et al., 2012) and consequently develop PTSD. In one study, 79\% of people with ID were exposed to at least one potentially traumatic event, with most exposed to multiple traumatic events $(M=2.8$ events; Scotti et al., 2012). Also, research by Wigham et al. (2011; 2014) shows that exposure to traumatic life events is associated with, and predicts, PTSD symptomology in people with ID.

Despite a greater incidence of exposure to traumatic life events and subsequent pathology in people with ID, the evidence for effective treatment options is limited. NICE (2005) recommends exposure therapies such as Trauma-focussed Cognitive Behaviour Therapy (TfCBT) and Eye Movement Desensitization Reprocessing (EMDR) as the two treatment options for PTSD in the general population. Nevertheless, only a small number of studies have been carried out investigating the use of various therapies for treating PTSD in people with ID and there is currently limited advice on the most effective treatment options. Previous studies include a small number of case studies describing the effectiveness of EMDR and psychodynamic therapy in reducing trauma-related symptoms (Mevissen and de Jongh, 2010; Mevissen et al., 2016; Jowett et al., 2016) and a pilot study of Tf-CBT reporting a 
medium effect size in reducing trauma-related distress for people with IDs (Stenfert-Kroese et al., 2016). Furthermore, Lemmon and Mizes (2002) in a case study reported that exposure therapy resulted in the elimination of distress to trauma-related stimuli, avoidance, anger outbursts and hypervigilance. More recently, in a descriptive case report of a young man with ID and a history of early traumatic event, it was reported that application of a positive behavioural support plan, incorporating behavioural therapies and medication, led to a reduction in challenging behaviours which were eventually extinguished whilst trauma-related symptoms also reduced (Langdon et al., 2017). Finally, a small case study on Compassionfocussed Therapy (CfT) found it a helpful approach for traumatic stress. However, there was little change on outcome measures (Cooper \& Frearson, 2017). This body of research suggests that psychological interventions can be potentially useful for treating traumatic symptoms and PTSD in people with IDs.

While psychological interventions for PTSD in people with ID appear to be promising (Vereenooghe and Langdon, 2013), to date there are no clinical guidelines regarding the treatment of PTSD in people with ID due to the lack of methodologically robust research studies. As a consequence, practitioners in ID health services who work with adults with ID who are also traumatised adapt and modify therapies validated for delivery to the general population (Barrowcliff \& Evans, 2015; Carrigan \& Allez, 2017). Thus, it is necessary to conduct a qualitative inquiry to explore how health professionals assess and treat trauma in this population, as this implicitly has important implications for future development of trauma services. The present study addressed this gap in the literature. 


\section{Aim}

The aim of this study was to explore the views of ID health care professionals on service provision for people with ID and traumatic stress.

More specifically the following questions were explored:

1. What are the ID health professionals' experiences of working with adults with ID and traumatic stress?;

2. How do ID health professionals think adults with ID and traumatic stress can be best supported within health services?;

3. What assessment and treatment options do ID health professionals use for traumatic stress in people with ID?;

4. What are the ID health professionals perceived barriers to treating traumatic stress in adults with ID? and

5. What are the needs for further support for both patients and clinicians?

\section{Method}

The data presented were derived from a qualitative phase of a larger pilot feasibility study examining the effectiveness of EMDR in adults with ID and DSM-5 PTSD (Karatzias et al., in press). Data were collected utilizing a semi-structured interview guide. Participants were either interviewed face-to-face in their place of work or by telephone.

\section{Participants}

A purposive sample of clinicians from specialist ID services was recruited. A total of 25 participants comprised of clinical psychologists $(n=7)$, psychiatrists $(n=6)$, nurses $(n=6)$ and allied health professionals and social workers $(n=6)$. Participants worked within four health service areas in Scotland, one Health and Social Care Trust in Northern Ireland and one Health 
Service Trust in England with an average of 11 years working in ID practice (range 1-30 years). The majority of participants, $72 \%(n=18)$ were female. Inclusion criteria were: clinicians working in a specialist ID service; experience of working with adults with ID and a history of traumatic life events; in post for at least one year; willing to consent to one-to-one face-to-face or telephone interview.

\section{Procedure and interview}

Recruitment occurred via specialist ID services. Subsequent to the identification of potential candidates, participants were provided with information sheets and consent forms prior to being contacted via phone by the researcher to ascertain their willingness to consent. Following consent, a suitable date and time for interview was arranged. Interviews were conducted via face-to-face and telephone interviews using a semi-structured interview schedule. The interview schedule was developed by the research team involving open-ended questions. The schedule enabled participants to share their practice experience as experts in the area and discuss issues relevant to the aims of the study (Dicicco-Bloom \& Crabtree, 2006). Interviews lasted for between 10 and 40 minutes (mean $=24$ minutes) and were audio-recorded and transcribed verbatim.

\section{Analysis and Rigour}

The data were analysed using thematic analysis, allowing for the analysis and identification of patterns and themes in qualitative data (Braun \& Clarke, 2013), in line with the scope of the present study. The current study used the six phases of thematic analysis proposed by Braun \& Clarke (2013). The first author read and reread the interview transcripts to achieve the first phase of 'familiarisation' of the data. The second phase, 'coding' involved collating and coding narrative accounts taken from the interviews. "Searching for themes' was achieved by looking for similarity between the codes and grouping similar codes together. The 
first two authors compared and discussed themes.' The themes that emerged in the analysis in relation to the coded extracts were retained andsimilar themes were collapsed together. On going analysis by the first two authors led to the 'defining and naming' of themes. Finally, the 'writing up' enabled authors to contextualise the findings in relation to the literature. To establish the credibility of themes, peer debriefing was performed throughout the research process to ensure that identified themes were consistent with the data and were not led by researcher expectations. Once consensus was reached, the themes were grouped by topics reflective of the research questions.

\section{Ethics}

Ethical approval was sought and granted by the Ethics and Governance Committee of the School of Health and Social Care (name removed for review) and the South East Scotland Research Ethics Committee and the Office for Research Ethics Committee Northern Ireland (ORECNI). Consent was obtained in writing from each participant in advance of the interview. Assurances were given that no identifiable information about the participants would be included in the study and that audio recordings would be deleted on completion of transcribing. 


\section{Findings}

Seven central themes were found, which have been grouped, reflecting each of the research questions. These will be described in turn sequentially and supported by narrative accounts.

\section{Theme 1: Unmasked traumatic symptoms}

The opening question to participants was "What are your experiences of working with traumatised adults with ID?" The central theme that emerged was unmasked traumatic symptoms. Participants identified a range of difficulties in assessing and treating both mental health problems and traumatic stress in adults with ID due to a fragmented referral system, often resulting from communication and cognitive limitations and diagnostic overshadowing. Traumatic stress was often not the primary reason for referral to specialist ID health services and appeared to go unreported and therefore untreated. Participants reported that possible traumatic stress was not routinely cited as one of the presenting reasons for referral. The reason often provided by the referrer was for assessment and management of behaviours such as anger, aggression and self-injurious behaviours. Traumatic events were not therefore routinely assessed by referring agencies and links made with the presenting clinical history. Traumatic events and subsequent distress were sometimes identified by the health professionals as part of the review of clinical histories and case records or part of the assessment process. In other cases, diagnosis was made following a disclosure by the adult with ID.

"I would say particularly the clients who I'm seeing who coming up for anger management or anxiety management, they all would have had some sort of trauma in their lives. And it's not maybe the focus of the treatment we're having but certainly I think would be part of the formulation that I am working with" 
"I have had people whereby they have been referred for anger management or challenging behaviour and then you realise actually the persons had a horrific childhood where they've been beaten and various other negative life events."

(12 Clinical Psychologist)

"Some of the people I worked with who didn't have a diagnosis, but had a lot of self-harm, sometimes they disclosed trauma, physical and sexual. I was working with them [person with ID] through anxiety management techniques and once we started working then a lot of the stuff came back and it turned out they had been abused and trauma was the real problem."

(10 Occupational Therapist)

\section{Theme 2: Trauma Informed Care}

There were two research questions aligned to service provision. The first was "How do you think people with ID and traumatic stress are best supported in services within health services?" Three themes emerged from this question. The first theme was about Trauma Informed Care (TIC). Most of the participants suggested a key feature of an effective trauma intervention for adults with ID is for services to be trauma-aware and focused by having in place care pathways, implemented by professionals with knowledge, skills and expertise to assess and treat adults with ID referred to services.

"It's about having trauma informed services certainly. So, people understand at least the basics about trauma and how the pathology presents, you know. Others don't and lots more needs to be done".

(09 Learning Disability Nurse) 
"I think care managers should continue with the service providers to help develop more of an understanding of what trauma is and how it can present differently in people with learning disabilities, particularly those who can't communicate as well, but how it should be on the agenda, but often isn't and gets missed."

(15 Clinical Psychologist)

\section{Theme 3: Person centred care and support}

The second theme to emerge from the question "How do you think people with ID and trauma are best supported in services within health services?" was Person centred care and support. Recognising the possible presence of psychological traumatic stress within adults with ID was an important element of adopting person-centred approaches to assessment and treatment. This was viewed as necessary, given the types of traumatic life events experienced and the different clinical presentations of adults with ID. Using assessments and adapting treatment approaches used for the general population was important in the delivery of care and treatment. Central to this was the need to work with other team members to collaborate on developing approaches that recognises the individual and their communication and support needs to ensure people can engage in therapy.

“I think it's really important that they're [treatment options] adapted to that person....it would definitely be individual. At the persons own pace as well, so giving them breaks if they want them."

(11 Social Worker)

"Having people who specialise in trauma treatment ... to actually treat people with learning disabilities who've been traumatised in the way that's most suitable for that individual. Rather than being tied to 'one size fits all' way of treating people."

(12 Clinical Psychologist) 
"Person-centred....they should be tailored to the patient...they've got to take account of the person's intellectual disability. They've got to be at the pace that the person can cope with. Communication issues are obviously going to be crucial, you know, all the descriptions have got to be at a level that the person can understand."

(14 Consultant Psychiatrist)

\section{Theme 4: Multi-disciplinary working}

Multi-disciplinary working was the primary theme to emerge from the second question aligned to service provision: "What assessment and treatments do you use when assessing and treating traumatic stress in adults with ID?" Multidisciplinary approaches to assessment and treatment were viewed by participants as central to effective care and support. The majority were of the view that a systemic, multi-disciplinary approach underpinned by a trauma-informed care pathway was necessary to ensure that assessment and treatment effectively addressed individual needs.

"A multidisciplinary approach is probably the best way because often it's about building relationship with the clients... a multidisciplinary approach where you know you've got a team working together and there's communication within the team, then at different points in that client's journey to kind of recovery from trauma, you've got maybe different people working who've got a different skill set, so that their needs can be met."

(19 Clinical Psychologist)

"I think having clinical psychologists in the team who have experience of working with people with trauma is really beneficial, so they can refer people within the team to different therapies. We have a music therapist as well so that can be really beneficial depending on the person."

(11 Social Worker) 


\section{Theme 5: Reasonable adjustments}

In response to the question "What assessment and treatments do you use when assessing and treating traumatic stress in adults with ID?" the emergent theme was Reasonable adjustments. Participants detailed the assessments and treatments options used with adults with ID. A wide range of treatments approaches were used including adapted CBT, counselling, EMDR, relaxation techniques, exposure therapy, systemic family therapy, mindfulness, music therapy, art therapy, storytelling and medication. Due to the limited validated assessment tools and lack of evidence-based treatments, participants drew on their clinical expertise to make reasonable adjustments to and modify existing mainstream assessment and treatments options to render them suitable for use with adults with ID.

"With exposure we would use maybe drawings instead of writing. Imagining is very difficult for some individuals so often it's the use of story or puppets, or just being imaginative. The therapists have to be quite imaginative, to find a way for the person to be able to, not only tell their story, but to re-enact some bits of it at times. ..The model is very much person-centred with the narrative very much being between, or within, the clinical setting. Between the patient and therapist if you like."

(07 Clinical Psychologist)

"It's about how that person is experiencing it (trauma)...Having a variety of resources that you can use. It might be that you have a toolbox of sensory stuff that is calming so if somebody is starting to feel that they are getting a bit tense you can try a variety of things.

(10 Occupational therapist) 
"I think it's important that they (assessments/treatments) are very individual. At the persons own pace as well, so giving them breaks if they want them. I've had to have very short sessions as well because they (the individual) can't focus for longer than maybe 20 minutes, half an hour tops or you've lost them. Recognising what people are interested in, what they like to do and sometimes its trial and error, it doesn't always work so it's going onto the next thing to try or having a break because it's maybe not the right time."

(11 Social worker)

\section{Theme 6: Barriers to treatment}

Participants were asked 'What are the main barriers and challenges to treating traumatic stress in adults with ID?' Barriers to treatment was the core theme reflective of this question. A number of barriers were reported by participants as inhibiting the delivery of therapy for adults with ID who have experienced trauma. These are arranged in three sub-themes. The first was related to therapeutic barriers regarding the availability of evidence-based, validated assessment and treatment options appropriate for the needs of adults with ID. The second was organisational barriers that focused on the need to develop awareness of the extent of and the presentation of traumatic stress in adults with ID. The third subtheme related to personal barriers intrinsic to adults with ID due to their cognitive impairment.

\section{Therapeutic barriers}

The lack of evidence-based treatment options for adults with ID resulted in a reliance on clinical skills, practice expertise and judgement. Often, standard treatment options used with the general population were adapted in the absence of evidence-based alternatives which was viewed as a significant barrier that required to be addressed. 
"I think the main challenges are sort of adapting things to meet the range of clients that we have. It's developing a range of therapies that we can use, because it still feels quite limited. I suppose from a point of view of efficacy, there is still limited evidence within our population, so you're sort of using stuff from general adult and tweaking it and change it, which is what we are used to doing."

(08 Clinical Psychologist)

"It's incredibly difficult for the smaller therapies, such as art therapy, to get the evidence-base growing."

(18 Music Therapist)

I think the problem with learning disability services, we try and do things, we try and kind of adapt what mainstream are doing. But I think we're really behind the times when it comes to trauma. And I think I can say that now having worked in a trauma service. I can really see that there are huge gaps in what we are doing with people with intellectual disabilities.

(19 Clinical Psychologist)

\section{Organisational barriers}

Healthcare professionals stated that there were training and continuing practice development barriers that need to be addressed within both mainstream and specialist ID services. Priority areas include increasing awareness and developing the knowledge and understanding of the presentation and treatment of traumatic stress in adults with ID. The organisational barriers were further compounded by issues related to staff turnover. Time constraints and lack of funding and resources were also viewed as a barrier to effective assessment and treatment.

"I think we could definitely all do with some more training in relation to different approaches that we could use....the staff that are supporting these people with intellectual disabilities, sometimes twenty-four-seven or on a daily basis, a lot of them have no experience of working 
with people with trauma, or if they have they're kind of guessing what's the right thing to do. Education and support is a big issue"

(11 Social Worker)

"It can be very time consuming in a way. That sounds very negative but the way that we would work with somebody might be quite a lot slower than in the general adult mental health arena where people are quicker to understand what they are doing, they do their homework between sessions or whatever, depending on what model you're using. But with our service users' things tend to happen a little bit more slowly because we're revisiting things, we're checking out their memory and assessing as we go."

(15 Clinical Psychologist)

\section{Personal barriers}

Personal barriers were identified in relation to the person with ID's, including the level of cognitive and communication impairment and additional supports required to engage in treatment. Participants also detailed barriers in delivering trauma therapy to those with more severe and profound ID.

“When you've got people with minimal verbalisation.... they struggle more with engaging in therapy."

(14 Consultant Psychiatrist)

"If they've got severe intellectual disability sometimes even using pictorial aids can be difficult if they can't express or find ways, or maybe they don't fully understand or able to relate to what are triggers for them, in that sense, and you would be looking on observation and other people to try and identify triggers."

(09 Learning Disability Nurse) 
"Some of the time, yes, I mean it works better for people with mild or moderate intellectual disabilities and obviously anyone whose borderline learning disabilities. They are all very capable of doing this, when you get to the lower end of the moderate intellectual disability severe and profound, you're suddenly very limited in what you can do because I wouldn't generally be working directly with the person they'd be working more systemically, they have more trouble seeing insight and remembering things."

(13 Clinical Psychologist)

\section{Theme 7: Awareness, training and education}

Participants were asked 'What are the needs for further support for both patients and clinicians?' The primary theme was 'awareness, training and education'. Participants acknowledged that in order to improve service provision for traumatic stress in adults with ID this would require 'staff training' and 'education' as well as 'supervision and support'. One psychologist noted:

"Mandatory training on trauma and the impact of trauma on the brain and on behavioural reactions and how that presents in people. I think there are some good training modules on trauma, but it's not necessarily mandatory for staff. And I think it should be.

(19 Clinical Psychologist) 


\section{Discussion}

This study aimed to explore the views of ID health care professionals on service provision for people with ID and psychological traumatic stress. The findings highlight that practitioners in specialist ID health services are routinely assessing and treating an increasing number of adults with ID who have experienced a range of traumatic life events, often with limited resources and a lack of evidence-based treatments options. The study findings highlight challenges in caring for traumatised people with ID, from the perspective of the service providers. Participants described trauma-informed care, person-centred care and support and multi-disciplinary care working as key features of services which best support people with ID who experience traumatic stress. In addition, a number of definitive barriers are highlighted by participants, which impact on the delivery of therapy for adults with traumatic stress and ID. The need for awareness, training and education, in order to improve service provision and meet the needs of this population, among participants was apparent. These findings were reflective of all participants as no differences were found among professionals and across countries.

In light of the findings, this study supports previous research regarding a fragmented referral system whereby health professionals may fail to consider the possibility of traumatic life events and PTSD at the point of referral as the cause of an individual's distress, particularly when presenting symptoms have endured for a prolonged period (Mitchell \& Clegg, 2005; Fletcher, 2016). Overshadowing, that is, attributing behavioural problems as part of ID itself is a well-known feature in mental health care for people with ID. A variety of problem behaviours commonly presented with PTSD, including aggression and anger outbursts, self-injurious behaviour, non-compliance, social isolation, sleeping problems, depression and restlessness can be easily attributed to one's ID. Assessment and diagnosis may be further compounded by psychiatric and physical comorbidities common in people with IDs (Cooper et al., 2007; Kwok 
and Chung 2007). There is clearly a need for further research on the phenomenology of traumatic stress in people with ID.

Our findings also echo previous research which suggested that it is common practice to make reasonable adjustments and modifications to existing treatment protocols to meet specific needs, including the needs related to ID and health (see Barrowcliff and Evans, 2015; Carrigan and Allez, 2016; Fernando and Medlicott, 2009; Marwood, Chinn, Gannon \& Scior, 2016). It was evident that whilst clinicians were usually able to make reasonable adjustments to enable adults with mild to moderate ID to engage in treatment, this was more difficult for those with more complex and severe ID and required alternative and more creative ways to ensure delivery of interventions due to deficits in the persons communication. The development of Trauma Informed Care (TIC) within healthcare services was also seen as the best way forward to support the development of trauma services specific to the needs of adults with ID. This supports the views of Kessler (2014) who recommends the delivery of TIC for organisations. Fallot and Harris (2009) discussed that TIC involves a cultural shift whereby safety, trustworthiness, choice, collaboration and empowerment are central to systemic functioning among service providers and service-users. The development of TIC within ID services would allow the use of interventions that consider the importance of trauma in clinical presentations and limit diagnostic overshadowing.

Previous studies have identified a number of barriers and challenges treating people with ID and mental health problems. A study by Marwood, Chinn, Gannon \& Scior (2018), which aimed to explore high-intensity therapist's experience of delivering CBT to individuals with ID in Improving Access to Psychological Therapies (IAPT) services, found that therapists lacked confidence in working therapeutically with people with ID and were uncertain about how to modify CBT. Consequently, they highlighted training and service development needs. 
Similarly, Shankland \& Dagnan (2015) who also explored IAPT practitioners' experiences of providing therapy to people with ID highlighted the need for extra training and flexibility in both the structure of therapy and the way it's delivered. In terms of the barriers and challenges to treating adults with ID and traumatic stress, it is not possible to compare the findings with previous research as this is the first study to explore these issues. The data confirm that the increasing demand for trauma therapy for people with ID has been met from practical and theoretical perspectives. Concerns include the perceived lack of appropriate training (Royal College of Psychiatrists, 2004) and theoretical concerns regarding whether people with IDs can experience mental illnesses, and assumptions that IDs is associated with cognitive limitations that prevent engagement in therapy (Adams and Boyd, 2010).

\section{Strengths and limitations}

The main strength of this study is that this is the first to report on the views and experiences of health professionals from multidisciplinary specialist ID health services of the delivery of psychological therapies for traumatic stress to adults with ID. The findings suggest that with adjustments and additional support, many adults with ID can access psychological therapies to assess and treat their traumatic stress. Moreover, the study has identified important issues of international relevance that need to be addressed to improve access to psychological therapies responsive to the needs of adults with ID and PTSD.

There are, however, a number of limitations to the study. The views of adults with an intellectual disability were not considered in this study. While attempts were made to ensure that the participants reflected the range of practitioners from specialist ID health services engaged in assessing and treating adults with ID who had experiences of treating traumatic stress, it is possible that their views and experiences are not reflected in other services internationally. Participants in this study were also drawn from services of three countries of 
the United Kingdom and are not necessarily representative of services elsewhere. Furthermore, although adequate for qualitative analysis, a small number of participants per discipline was included in our study.

\section{Directions for future research}

We propose five areas for future research. The first is, in order to enable an accurate diagnosis in adults with ID and access to appropriate treatments, development of populationspecific PTSD diagnostic assessments that correspond to DSM-5 criteria should be developed. Whilst there are limited evidence-based validated assessment tools with good psychometric properties, such as the Lancaster and Northgate Trauma Scale (LANTS) (Wigham, Hatton \& Taylor, 2011) and the Impact of Event Scale-Intellectual Disabilities (IES-IDs) to assess PTSD in adults with ID (Hall, Jobson \& Langdon, 2014), further research is required to adapt and validate assessments tools that are sensitive in identifying traumatic stress in line with DSM-5 and ICD-11 criteria.

Secondly, there is a small number of randomised controlled trials (RCTs) regarding the efficacy of psychological therapies for adults with ID, albeit for those with mild to moderate ID, however these are not focused on traumatic stress, but rather other psychological concerns. Previous research in the area suffered a number of limitations including inadequate numbers of participants and poor design (Oliver et al., 2002; 2003; Sturmey et al., 2004). Therefore, there is the need for large-scale, high quality RCTs exploring both the efficacy and effectiveness of psychological therapies for adults with ID, across all severity levels of ID, and PTSD. These studies must also include direct comparisons with evidenced-based alternative interventions such as pharmacological interventions and other forms of psychological therapy. Over time, a larger evidence-base will contribute to the development of clinical guidelines on how best to support trauma in adults with ID. 
Thirdly, it is evident that further work is required on education and practice development needs. People with IDs and their families need access to education about the presentation of traumatic stress and where to access advice, help and support. As misinterpretation of trauma symptomatology such as challenging behaviour may be detrimental to people with ID, this can be prevented by staff training (Grey, McClean and Barnes-Holmes (2002). At a continuing professional development (CPD) level, access to education for practitioners across health and social care services and care providers need to be provided to improve their knowledge and understanding of the presence and presentation of traumatic stress in adults with ID (Embregts et al., 2017). Raising awareness of traumatic stress is also required by professionals in primary care and by people with ID, their families and carers, so that it can be signposted as part of the referral process to specialist ID health services (Kessler et al., 2014). Fourthly, conducting qualitative interviews with people with ID who have undergone trauma to obtain an insight into their experiences of the support services received to treat their condition.

Finally, how the presentation of PTSD and traumatic stress more generally in people with ID differs from the general population is an area requiring further investigation. At the moment, there is an assumption that traumatic stress manifests similarly in both the general population and the ID population. It might well be the case that common presenting symptoms in people with ID, such as challenging behaviour, might follow exposure to traumatic events. It is only when we know how trauma manifests in people with ID, that we will be able to develop effective treatments and provide efficient service delivery. Ultimately, this will enable the appropriate application of principles of TIC. 


\section{Conclusion}

This study has explored the experiences of specialist ID health professionals assessing and treating traumatic stress in adults with ID. Despite the lack of rigorous evidence for effective psychological interventions for PTSD in adults with ID, health care professionals are continuing to treat adults with traumatic stress and ID by adjusting evidence-based psychological interventions and resources. There is a need to develop an evidence base in the treatment of traumatic stress in people with IDs. Raising awareness through the implementation of TIC within healthcare services is recommended as the best way forward in trauma service development. 


\section{References}

American Psychiatric Association (2013) The Diagnostic and Statistical Manual of Mental Disorders: (5 $5^{\text {th }}$ Edition). American Psychiatric Association: Washington, DC.

Barrowcliff, A.L. \& Evans, G.A.L. (2015). EMDR treatment for PTSD and intellectual disability: A case study. Advances in Mental Health and Intellectual Disabilities, 9(2), 90-98.

Braun, V. \& Clarke, V. (2013). Successful qualitative research: A practical guide for beginners. London: Sage.

Carrigan, N. \& Allez, K. (2017). Cognitive Behaviour Therapy for Post-Traumatic Stress Disorder in a person with an Autism Spectrum Condition and Intellectual Disability: A Case Study. Journal of Applied Research in Intellectual Disabilities, 30(2), 326-335.

Cooper, R. and Frearson, J. (2017) Adapting compassion focused therapy for an adult with a learning disability —A case study. British Journal of Learning Disabilities 45: 142-150.

Cooper, S-A., Smiley, E., Morrison, J., Williamson, A. \& Allan, L. (2007) Mental ill-health in adults with intellectual disabilities: prevalence and associated factors. The British Journal of Psychiatry, 190, 27-35.

DiCicco-Bloom, B., \& Crabtree, B. F. (2006) The qualitative research interview. Medical Education, 40(4), 314-321. 
Fallot, R. and Harris, M. (2009) Creating cultures of trauma-informed care (CCTIC): a selfassessment and planning protocol. Washington DC: Community Connections.

Fletcher, R.J., Barnhill, J., McCarthy, J., Strydom, A. (2016) From DSM to DM-ID. J Ment Health Res Intellect Disabi. 9(3):189-204.

Fernando, K., \& Medlicott, L. (2009). My shield will protect me against the ANTS: Treatment of PTSD in a client with an intellectual disability. Journal of Intellectual and Developmental Disability, 34(2), 187-192.

Gilderthorp, R.C. (2015) Is EMDR an effective treatment for people diagnosed with both intellectual disability and post-traumatic stress disorder? J Intellect Disabil. 19(1): 58-68.

Grey, I.M., McClean, B., \& Barnes-Holmes, D. (2002) Staff attributions about the causes of challenging behaviours: Effects of longitudinal training in multi-element behaviour support. Journal of Learning Disabilities, 6(3), 297-312.

Horner-Johnson, W. and Drum, C.E. (2006) Prevalence of maltreatment of people with intellectual disabilities: a review of recently published research. Mental Retardation and Developmental Disabilities Research Review 12: 57-69.

Hughes, K., Bellis, M.A., Jones, L., et al. (2012) Prevalence and risk of violence against adults with disabilities: a systematic review and meta-analysis of observational studies. The Lancet 379: $1621-1629$.

Hatton, C. (2002) Psychosocial interventions for adults with intellectual disabilities and mental health problems: A review, Journal of Mental Health, 11:4, 357-374. 
Jones, L., Bellis, M.A., Wood, S., et al. (2012) Prevalence and risk of violence against children with disabilities: a systematic review and meta-analysis of observational studies. The Lancet 380: 899-907.

Jowett, S., Karatzias, T., Brown, M., Grieve, A., Paterson, D. \& Walley, R. (2016) Eye Movement Desensitization and Reprocessing (EMDR) for DSM-5 Posttraumatic Stress Disorder (PTSD) in adults with intellectual disabilities: A case study review. Psychological Trauma, Theory, Research, Practice, and Policy, 8(6), 709.

Keesler, JM. (2014) A call for the integration of trauma-informed care among intellectual and developmental disability organizations. Journal of Policy and Practice in Intellectual Disabilities 11: 34-42.

Kwok, H. \& Cheung, P.W.H. (2007) Co-morbidity of psychiatric disorder and medical illness in people with intellectual disabilities. Current Opinion in Psychiatry $20443-49$.

Langdon PE, Dalton D, Brolly K, et al. (2017) Using positive behavioural support as a treatment for trauma symptoms with a man with intellectual disabilities. International Journal of Positive Behavioural Support 7(1): 31-37.

Leeb, R.T., Bitsko, R.H., Merrick, M.T., et al. (2012) Does childhood disability increase risk for child abuse and neglect? Journal of Mental Health Research in Intellectual Disabilities 5: 4-31.

Lemmon, V.A. and Mizes, J.S. (2002) Effectiveness of exposure therapy: A case study of posttraumatic stress disorder and mental retardation. Cognitive and Behavioral Practice, 9 (4), pp. 317-323. 
Marwood, H., Chinn, D., Gannon, K. and Scior, K. (2018) The experiences of high intensity therapists delivering cognitive behavioural therapy to people with intellectual disabilities. $J$ Appl Res Intellect Disabil, 31, 76-86.

Mevissen, L. \& de Jongh, A. (2010) PTSD and its treatment in people with intellectual disabilities: A review of the literature. Clinical Psychology Review, 30, 308-316.

Mevissen, L., Didden, R. \& de Jongh, A. (2016) Assessment and treatment of PTSD in people with intellectual disabilities. Springer.

Mitchell, A., \& Clegg, J. (2005) Is Post-Traumatic Stress Disorder a helpful concept for adults with intellectual disability?. Journal of Intellectual Disability Research, 49(7), 552-559.

National Institute of Health and Clinical Excellence (NICE) Guidelines (2005) Post -traumatic stress disorder: The management of PTSD in adult and children in primary and secondary care. London, UK: Gaskell and the British Psychological Society.

Scotti, J.R., Stevens, S.B., Jacoby, V.M., Bracken, M.R., Freed, R. \& Schmidt, E., (2012) Trauma in people with intellectual and developmental disabilities: Reactions of parents and caregivers to research participation. Intellectual and developmental disabilities, 50(3), 199206.

Shankland, J. \& Dagnan, D. (2015) IAPT practitioners' experiences of providing therapy to people with intellectual disabilities. Advances in Mental Health and Intellectual Disabilities, 9(4), 206-214. 
Stenfert-Kroese, B., Stenfert-Kroese, B., Willott, S., Willott, S., Taylor, F., Taylor, F., Smith, P., Smith, P., Graham, R., Graham, R. \& Rutter, T. (2016) Trauma-focussed cognitivebehaviour therapy for people with mild intellectual disabilities: Outcomes of a pilot study. Advances in Mental Health and Intellectual Disabilities, 10(5), 299-310.

Vereenooghe, L. \& Langdon, P. E. (2013). Psychological therapies for people with intellectual disabilities: A systematic review and meta-analysis. Research in Developmental Disabilities, 34(11), 4085-4102.

Wigham, S., Hatton, C. and Taylor, J.L. (2011) The effects of traumatizing life events on people with intellectual disabilities: a systematic review. Journal of Mental Health Research in Intellectual Disabilities 4(1): 19-39.

Wigham, S., Taylor, J.L. and Hatton, C. (2014) A prospective study of the relationship between adverse life events and trauma in adults with mild to moderate intellectual disabilities. Journal of Intellectual Disability Research 58: 1131-1140. 